OPEN ACCESS

\section{First experiments with the polarized internal gas} target at ANKE/COSY

To cite this article: M Mikirtychyants et al2011 J. Phys.: Conf. Ser. 295012148

View the article online for updates and enhancements.

\section{Related content}

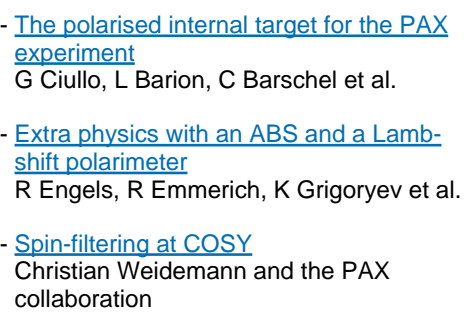

The polarised internal target for the PAX experiment

G Ciullo, L Barion, C Barschel et al.

Extra physics with an ABS and a Lambshift polarimeter

$\mathrm{R}$ Engels, $\mathrm{R}$ Emmerich, $\mathrm{K}$ Grigoryev et al.

- Spin-filtering at COSY

Christian Weidemann and the PAX collaboration

\section{Recent citations}

The neutron-proton charge-exchange
amplitudes measured in the dp ppn
reaction
D. Mchedlishvili et al

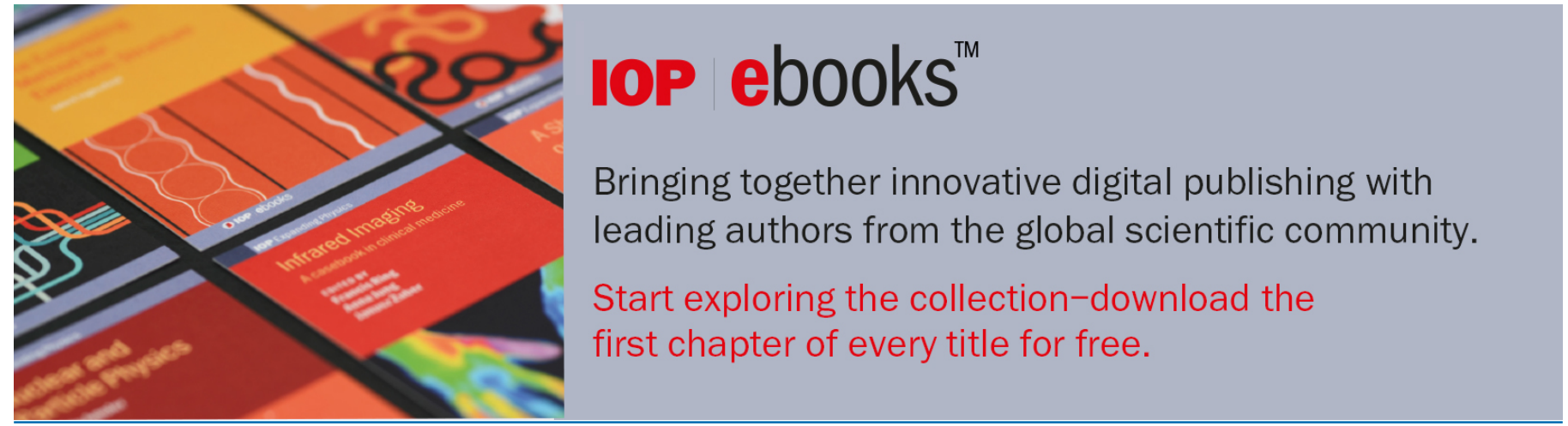

This content was downloaded from IP address 46.78 .228 .200 on 30/06/2020 at 07:12 


\title{
First experiments with the polarized internal gas target at ANKE/COSY
}

\author{
M Mikirtychyants ${ }^{1,2}$, R Engels ${ }^{2}$, K Grigoryev ${ }^{1,2}$, A Vasilyev $^{1}$, D Chiladze ${ }^{2}$, \\ A Kacharava ${ }^{2}$, S Mikirtychyants ${ }^{1,2}$, F Rathmann ${ }^{2}$, J Sarkadi ${ }^{2}$, R Schleichert ${ }^{2}$,

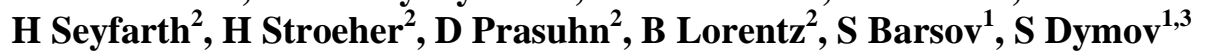 \\ ${ }^{1}$ Petersburg Nuclear Physics Institute, Gatchina, Russia \\ ${ }^{2}$ Institute for Nuclear Physics / JCHP, Forschungszentrum Jülich, Germany \\ ${ }^{3}$ Laboratory of Nuclear Problems, Joint Institute for Nuclear Research, Dubna, Russia \\ E-mail: m.mikirtychyants@fz-juelich.de
}

\begin{abstract}
A Polarized Internal gas Target (PIT) has been developed for the ANKE spectrometer at COSY. After its first installation at the ANKE target position in summer 2005, commissioning studies were carried out. In March 2006, first single polarization measurements with the polarized hydrogen beam from an Atomic Beam Source (ABS) were performed. The beam was injected into a storage cell made from aluminum foil. The data analysis showed that the events from the extended gas target can be clearly identified in the ANKE forward detection system. Using unpolarized nitrogen, the background from the cell walls could be determined as well. The thickness of the gas in the storage cell with the hydrogen atoms in hyperfinestate $|1\rangle$ was measured as $2 \times 10^{13}$ atoms $/ \mathrm{cm}^{2}$. The ABS jet target thickness was $1.5 \times 10^{11}$ atoms $/ \mathrm{cm}^{2}$. In November 2006, the commissioning of a Silicon Tracking Telescope (STT) was successfully finished. In the following beam time in January 2007, a new storage cell from aluminum coated with teflon was used together with the STT. The Lamb-shift polarimeter (LSP) was mounted below the target chamber to allow for online tuning of the transition units and monitoring of the ABS jet polarization. A first doublepolarized experiment was performed, the results will be presented.
\end{abstract}

\section{Introduction}

In 2004 the Atomic Beam Source (ABS) [1] and the Lamb-shift polarimeter (LSP) [2] were transferred from the laboratory to the COSY building. After all necessary tests, where the parameters, listed in Table 1, were determined, in the summer of 2005 the source was installed at the spectrometer ANKE for further commissioning. Measurements to determine the COSY-beam dimensions at the ANKE-target position and first tests with storage-cell prototypes were carried out in parallel to these studies.

Table 1. Main parameters of the polarized atomic beam source of ANKE/COSY.

\begin{tabular}{|c|c|c|c|}
\hline Gas Type & Intensity, at/s & $\mathrm{P}_{\mathrm{z}}$ & $\mathrm{P}_{z z}$ \\
\hline \multirow{2}{*}{ Hydrogen } & \multirow{2}{*}{$(7.8 \pm 0.2) \times 10^{16}$} & $+0.89 \pm 0.01$ & - \\
\cline { 3 - 4 } & & $-0.96 \pm 0.01$ & - \\
\hline Deuterium & $(3.9 \pm 0.1) \times 10^{16}$ & +0.73 & +0.77 \\
\hline
\end{tabular}




\section{Polarized internal target at ANKE}

In order to achieve the maximum luminosity in the experiments with the internal gas target it is important to minimize the dimensions of the storage-cell tube. During the first test in February 2004 the diameter of the COSY beam at injection and after acceleration at the ANKE target position was measured. For this, a frame carrying various diaphragms was constructed. The diaphragm, which was

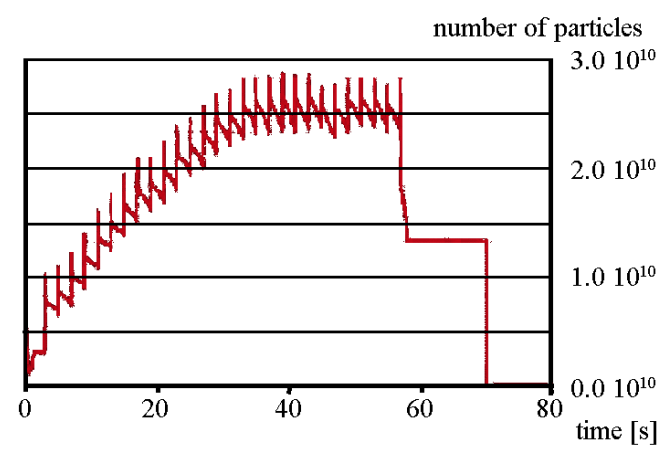

Figure 1. The beam-current transformer converted to the number of the stored protons in the COSY ring during stacking (28 stacks) through a storage cell $\left(20 \times 20 \times 400 \mathrm{~mm}^{3}\right)$ followed by $2 \mathrm{~s}$ of electron cooling and accelerating to flat top energy.

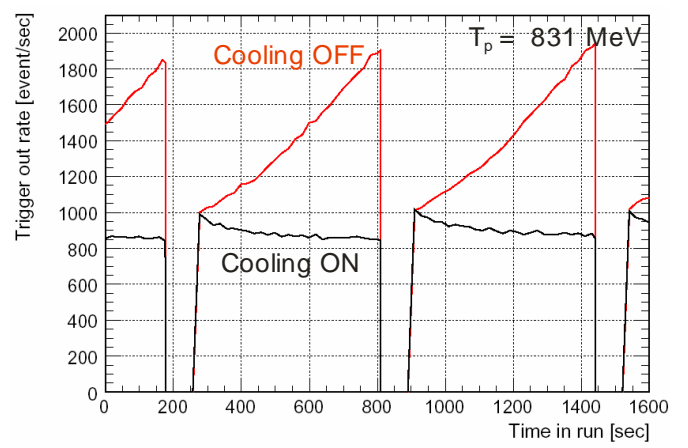

Figure 2. The trigger rate during data taking with stochastic cooling switched on and off during a set of cycles. The strong increase of the trigger rate at the flat top energy after acceleration with stochastic cooling off occurs due to the increase of beam-cell wall interactions, caused by beam heating.

mainly used, had dimensions of $50_{\text {hor }} \times 25_{\text {vert }} \mathrm{mm}^{2}$, i.e. larger than the expected beam size. During the tests, the supporting frame was moved across the beam by stepper motors. First, the center of the diaphragm was placed at the expected center of the COSY beam. By moving the diaphragm, the COSY beam current was gradually decreased and the beam full size could be measured ${ }^{1}$. At injection, the beam had elliptical shape and its full size was $38_{\text {hor }} \times 17_{\text {vert }} \mathrm{mm}^{2}$. The accelerated beam without target had a size of $9_{\text {hor }} \times 14_{\text {vert }} \mathrm{mm}^{2}$. With the cluster-target beam (density: $10^{12}$ atoms $/ \mathrm{cm}^{2}$ ) it increased to $17_{\text {hor }} \times 17_{\text {vert }} \mathrm{mm}^{2}$ due to beam heating by the target [3].

In addition to the determination of the cell dimensions, special care was taken to shield various ABS components (e.g., vacuum pumps) against strong stray field of the central ANKE dipole D2. This issue is especially important for the weak-field transition unit (WFT), which is located about $400 \mathrm{~mm}$ away from the D2 gap.

\section{Polarized internal target commissioning}

Based on the measured results, two storage-cell prototypes were built from a $25 \mu \mathrm{m}$ thick aluminum foil $(99.95 \mathrm{Al})$. With acceleration of an unpolarized deuteron beam through the large cell $\left(30_{\text {hor }} \times 20_{\text {vert }} \mathrm{mm}^{2}\right)$ to an energy of about $2.1 \mathrm{GeV}$, it was possible to store and accelerate more than $2 / 3$ of the injected deuterons $\left(\sim 9 \times 10^{9}\right)$ into the COSY ring. Using beam scrapers in the opposite section of the accelerator ring, the dimensions of the stored beam in the cell were decreased to $13_{\text {hor }} \times 11_{\text {vert }} \mathrm{mm}^{2}$. With a small cell of $15_{\text {hor }} \times 15_{\text {vert }} \mathrm{mm}^{2}, 1.7 \times 10^{9}$ deuterons, i.e., about $15 \%$ of the injected deuterons, were successfully stored in the COSY ring. The length of both cells was $220 \mathrm{~mm}$.

For the first beamtime an aluminum foil, covered with PTFE to minimize depolarization on the surface, was used for the new prototype of the storage cell. The beam tube of this prototype was

\footnotetext{
${ }^{1}$ These measurements were done with a $2.1 \mathrm{GeV}$ proton beam without using any cooling procedures (electron cooling at injection or stochastic cooling after acceleration) and stacking procedure at injection.
} 
$400 \mathrm{~mm}$ long and had a cross section of $20_{\text {hor }} \times 20_{\text {vert }} \mathrm{mm}^{2}$. During the run, stacking injection [4] and electron cooling were employed to increase the number of stored and accelerated protons with the storage cell in place (Fig. 1). As a last step, the ANKE spectrometer magnet D2 was moved to the position which corresponds to a deflecting angle of $9.2^{\circ}$ for the first beam bending magnet D1. In this configuration, $6.4 \times 10^{9}$ protons could be stored and accelerated in the ring. This corresponds to about $50 \%$ of the number of particles which can be accelerated without cell and stacking at injection. This number yields an appreciable luminosity of about $10^{29} \mathrm{~cm}^{-2} \mathrm{~s}^{-1}$ for double polarization experiments.

For beam energies higher than $831 \mathrm{MeV}$ stochastic cooling can be used at COSY. This will compensate for the beam heating by the target. In Fig. 2 the total trigger rate during data acquisition is shown as a function of time during different beam cycles. Without stochastic cooling being employed, beam heating leads to an intensive interaction of beam halo and thus extensive background growth.

In addition to the storage cell tests, the ABS beam was used as a jet target. In a first experiment the target position along the COSY beam direction could not be identified by the ANKE detector system due to very high rest gas pressure. For a second experiment an ABS beam cryo catcher was built and installed below the interaction point with the COSY beam. This allowed us to improve the pressure in the target chamber by one order of magnitude to $3.7 \times 10^{-8}$ mbar. With use of vertex reconstruction, the jet-target position could be clearly identified. The measured integral jet-target thickness of $\sim 1.5 \times 10^{11} \mathrm{~cm}^{-2}$ perfectly matches the predicted value.

\section{Results of the commissioning}

In early 2007, the LSP was used to tune and to control the polarization of the ABS beam. However, its use in the strong magnetic stray field of the spectrometer magnet D2 revealed a number of problems. First of all, it is the reduced sensitivity of the LSP due to the deflection of the slow protons behind the Glavish-type ionizer. Second, the deflection of the quantization axis (longitudinal solenoid field of the ionizer) due to superpositioning with the stray field leads to a "magnetic misalignment" of the LSP, which could not be compensated by the installed Wien-filter. This resulted in an underestimation of the measured polarization ${ }^{2}$ and, furthermore, in a wrong sign of the vector polarization. Nevertheless, the transition units could be tuned and the polarization, which was measured once per day, could be controlled and was found to be stable within 5\% during one week of operation.

During this beam time, a storage cell $\left(20_{\text {hor }} \times 20_{\text {vert }} \times 380 \mathrm{~mm}^{3}\right)$ was used. In addition, $\mathrm{H}_{2}$ and $\mathrm{N}_{2}$ could be injected into the cell by two separate gas feeding tubes. A first silicon tracking telescope (STT) was mounted around the cell. Polarized or unpolarized deuterons were accelerated to the flattop energy of $\mathrm{T}_{\mathrm{d}}=1.2 \mathrm{GeV}$ through the storage-cell tube filled with polarized hydrogen from the ABS or with unpolarized $\mathrm{H}_{2}$ or $\mathrm{N}_{2}$ gas from the calibrated gas supply system.

Fig. 3 shows sample spectra from the elastic scattering $\vec{d} \vec{p}$ (upper panels) and the $\vec{d} \vec{p} \rightarrow\left(d p_{s p}\right) \pi^{0}$ reaction (lower panels). Both missing-mass spectra could be corrected for events, which are produced at the cell walls. For this reason, data were taken with $\mathrm{N}_{2}$ in the storage cell. The $\mathrm{N}_{2}$-target density was adjusted in a way to provide the same COSY beam heating as for the $\vec{H}$ [5]. The event distributions of these runs were subtracted from the original measured spectra with hydrogen in the cell, and the results are shown on the right-hand side of Fig. 3.

The analyzing powers for the reaction $\vec{d} \vec{p} \rightarrow\left(d p_{s p}\right) \pi^{0}$ for different scattering angles are known with good precision, see ref. [6], and therefore, the polarization of the storage-cell target was determined with an unpolarized deuteron beam at COSY as $\mathrm{P}_{\mathrm{y}}=0.79 \pm 0.07$ based on the measured asymmetries. Vice versa, the polarization of the COSY beam can be observed with unpolarized hydrogen gas in the storage cell as well.

\footnotetext{
${ }^{2}$ The measured polarization was about $22 \%$ of the expected value.
} 

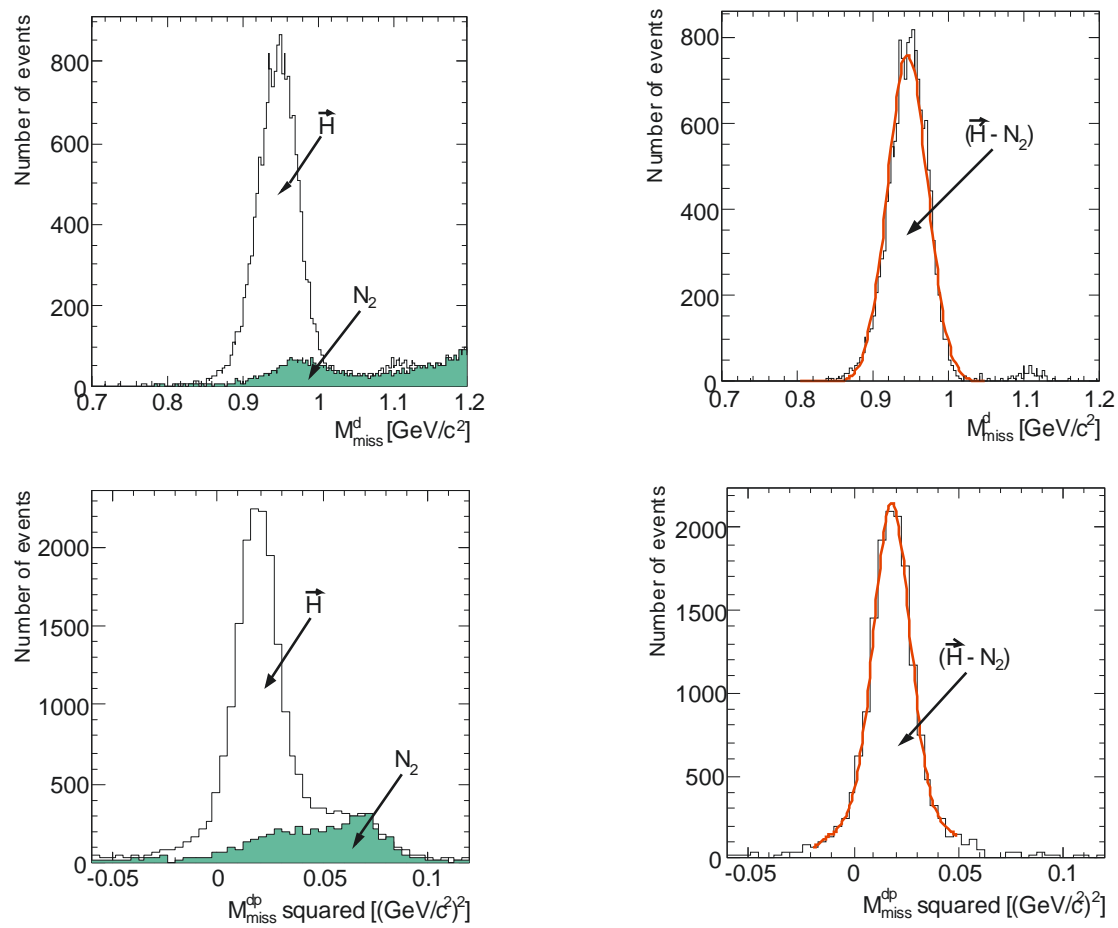

Figure 3. Missing-mass spectra for the proton from elastic $\vec{d} \vec{p}$ scattering (upper panels) and the $\pi^{0}$ from the quasi-free $n p \rightarrow d \pi^{0}$ reaction (lower panels) before and after background substraction (on the left- and right-hand side, respectively). The background substraction is based on the additional measurement with $\mathrm{N}_{2}$ gas in the target cell.

\section{Latest results and outlook}

In the fall of 2009 a long beam time at ANKE on the double-polarized $\vec{p} \vec{d}$ breakup reaction was performed at flat top energies $\mathrm{T}_{\mathrm{d}}=1.2$ and $\mathrm{T}_{\mathrm{d}}=2.23 \mathrm{GeV}$ [7]. However, a modified LSP with a rotatable Wien filter was not available to compensate for the deflection of the quantization axis by the magnetic stray fields of the ANKE spectrometer magnet. Furthermore, measured value of the positive target polarization was found to be about $10 \%$ smaller compared to the previous result obtained during the commissioning run. In order to overcome these problems in coming experiments a substantial improvement of the magnetic shielding of the Wien filter and the electrostatic deflector, as well as compensation coils in the ABS weak-field transition unit are under preparation.

\section{References}

[1] Rathmann F et al, Proc. 15th Int. Spin Physics Symposium, Upton, NY, USA, 2002. AIP Conf. Proc. 675 (2003) 553.

[2] Engels R et al, Rev. Sci. Instrum. 74 (2003) 4607.

[3] Grigoryev K et al, Nucl. Instr. and Meth. A599, 130 (2009)

[4] Stein H J et al, Proc. 18th Conf. on Charged Particle Accelerators (RUPAC 2002), Obninsk, Russia, 2002. I.N. Meshkov (Ed. in chief), NRCRF Obninsk (2004) 220.

[5] Rathmann F et al, Phys. Rev. C 58, 658 (1998).

[6] Chiladze D, Phys. Rev. ST-AB, 9, 050101 (2006).

[7] COSY Proposal No. 172, Spokespesons: A. Kacharava, F. Rathmann and C. Wilkin, http://www.fZ-juelich.de/ikp/anke/en/proposals.shtml 